\title{
Nueva localidad y gradiente altitudinal de Oedipina taylori (Stuart, 1952) (Caudata: Plethodontidae) en El Salvador
}

\author{
New location and gradient altitudinal of Oedipina taylori (Stuart, 1952) \\ (Caudata: Plethodontidae) in EI Salvador
}

\author{
Emanuel Stanley Morán Hidalgoㅁ, Vladlen Ernesto Henríquez², \\ Néstor Esaú Ruballo Marroquin ${ }^{3}$
}

\begin{abstract}
Resumen
Se da a conocer la nueva localidad y gradientes altitudinales de la especie Oedipina taylori en El Salvador. Se reporta la nueva ubicación para el Área Natural Protegida Río Sapo, departamento de Morazán, y se amplía su nueva distribución altitudinal en el Área Natural Protegida Parque Walter Thilo Deininger, departamento de La Libertad y Parque Nacional El Imposible, departamento de Ahuachapán.

Palabras clave: Altitudinal, Gradiente, Nuevo, Registro, Salamandra.

Abstract

This research discloses the new location and altitudinal gradients for the species Oedipina taylori in El Salvador. We report the new location for the Rio Sapo Natural Protected Area, Morazán department, and its new wide altitudinal distribution in the Natural Protected Area Park Walter Thilo Deininger, department of La Libertad and El Imposible National Park, Ahuachapan department.
\end{abstract}

Keywords: Altitudinal, Gradient, New, Record, Salamander.

La diversidad de Salamandras, en los trópicos del nuevo mundo ha aportado un gran número de especies, que son diversas en morfología y ecología (Wake y Good 1997). El género Oedipina se distribuye desde el sur de México hasta el noroeste de América del Sur. Las especies del género Oedipina se diferencian del resto de salamandras neotropicales debido a sus cuerpos alargados y extremadamente delgados, extremidades pequeñas y colas muy largas (Köhler 2011).

Las especies de Oedipina usualmente son confusas y similares en estructura morfológica y coloración, de color gris a marrón a excepción de algunas especies que poseen patrones dorsales pigmentados, sobre todo en la cabeza (Wake y Good
1997), reportando actualmente 35 especies para Centro América (Brame 1968, Köhler 2011, McCranie y Townsend 2011, Sunyer et al. 2011, Boza Oviedo et al. 2012, Brodie et al. 2012). El Salvador posee un solo representante Oedipina taylori (Köhler et al. 2006).

Los miembros del género Oedipina se caracterizan por su falta de diferenciación morfológica y análisis filogenético (Taylor 1952, Brame 1968, Good y Wake 1997, García-Paris y Wake 2000). Esto puede ser una explicación a que algunos autores los consideran en la lista de especies del género Oedipina a O. salvadorensis (Rand 1952).

Según (Rand 1957) existe la necesidad de aclarar las relaciones de las otras especies encontradas

\footnotetext{
Escuela de Biología, Facultad de Ciencias Naturales y Matemática, Universidad de El Salvador. e-mail: stanley_hidalgo@yahoo.com

2 Biólogo y Técnico en Sistemas de Información Geográfica. World Institute for Conservation and Enviroment. e-mail: vladlen.henriquez@birdlist.org

3 Escuela de Biología, Facultad de Ciencias Naturales y Matemática, Universidad de El Salvador. e-mail: ruballonestor@yahoo.com Fecha recepción: Febrero 18, 2013 Fecha aprobación: Mayo 2, 2013
} 
en la vertiente del Pacífico O. ignea y O. taylori (Stuart 1952), con el hallazgo de O. salvadorensis, en el mismo año. Brame (1968) analizó 14 individuos incluyendo el holotipo de O. salvadorensis, colectados en El Salvador, determinando que son $O$. taylori, conservando a $O$. salvadorensis en sinonimia de O. taylori (Brodie y Campbell 1993).

O. taylori, se registra a elevaciones moderadas desde los 140-1140 msnm (Köhler 2011). Su distribución geográfica es en la vertiente del Pacífico, desde el sureste de Guatemala, a lo largo del sur de Honduras hasta el centro de El Salvador; también se ha registrado en la parte superior del Valle del Río Motagua en las proximidades de Doña María, Guatemala (Köhler et al. 2006; McCranie y Castañeda 2007). Su distribución ecológica abarca los ecosistemas de bosque tropical deciduo, bosque tropical semideciduo, bosque tropical siempreverde, bosque ripario, zonas de plantación de café y además se reportan en el área urbana (Köhler et al. 2006, Programa REDD CCAD GIZ 2011).
Estos nuevos registros, contribuyen en el conocimiento de la distribución de los anfibios en El Salvador, ya que está especie se encuentra en estado de «amenazada» en el país (MARN 2009), debido a la pérdida de hábitat natural y la creencia popular de confundirla con una serpiente. Según Acevedo et al. (2010), posee un estatus de menor preocupación (LC) a nivel mundial, a pesar que se desconoce su población actual.

Las nueva localidad de O. taylori, se registra en el Área Natural Protegida Río Sapo, en el mes de septiembre del 2012, ubicada en el municipio de Arambala, departamento de Morazán (Figura 1), que forma parte del Área de Conservación Nahuaterique, una zona fronteriza con la República de Honduras (MARN 2011). En la zona se desarrollan los ecosistemas de bosque tropical semideciduo mixto submontano, bosque tropical semideciduo mixto montano inferior y sabana de graminoides cortos con árboles latifoliados siempreverdes de variedad Curratella americana (Centro de Estudios Ambien-

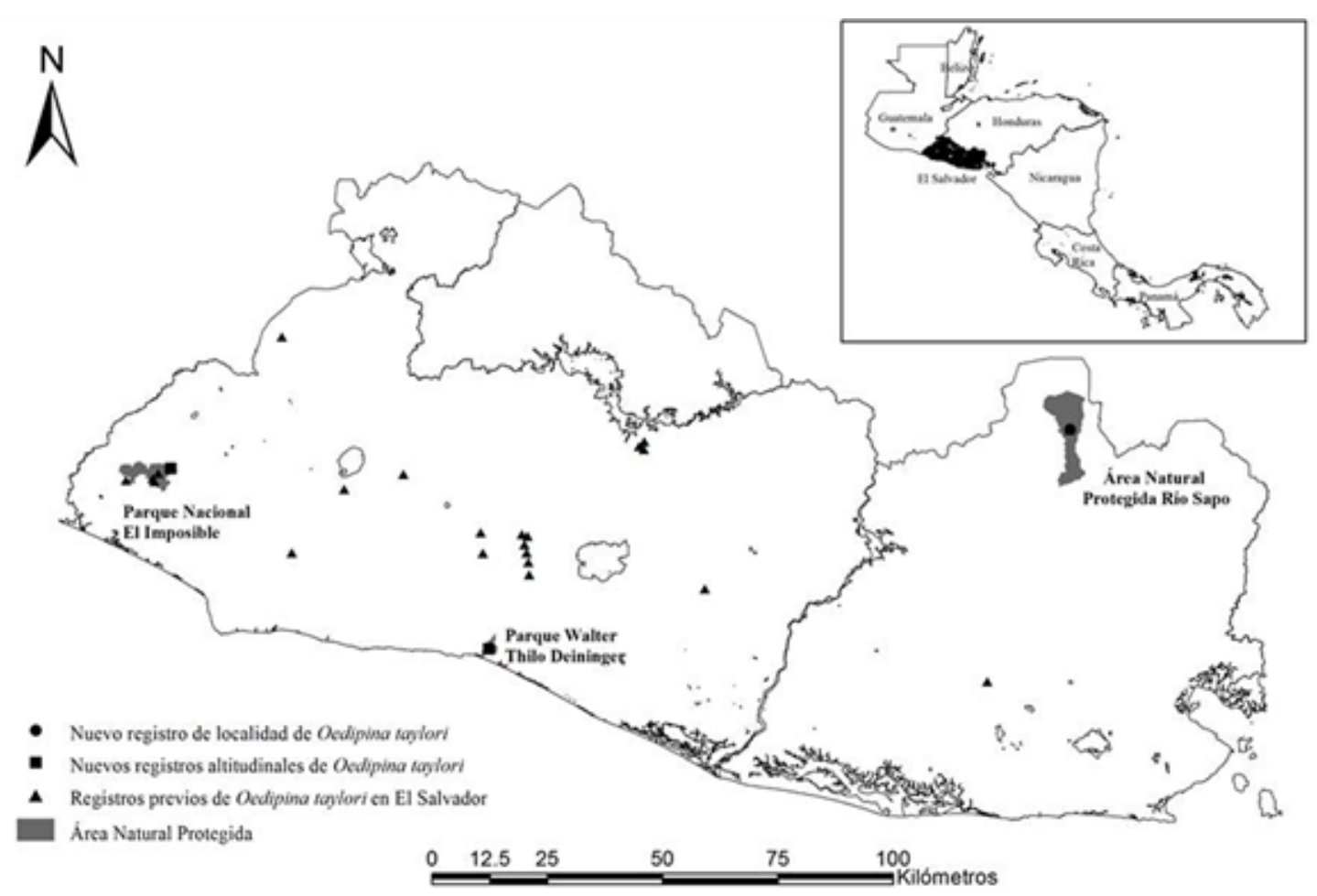

Figura 1. Ubicación de la nueva localidad de Oedipina taylori para El Salvador en el Área Natural Protegida Río Sapo (Circulo), nuevos registros altitudinales en el Área Natural Protegida Parque Walter Thilo Deininger (Cuadro), municipio y departamento de La Libertad; Parque Nacional El Imposible, municipio de Tacuba, departamento de Ahuachapán (Cuadro), registros previos de O. taylori en El Salvador (Triángulo). Julio de 2007, agosto y septiembre de 2012 y registros anteriores de la especie en El Salvador. 
tales y Biodiversidad et al. 2009, Fichas de Áreas de Conservación 2011).

Anteriormente se han realizaron inventarios de anfibios y reptiles en el Área Natural Protegida Río Sapo (Leenders y Watkins-Collwel 2004), no obstante, no se reporta la ocurrencia de la especie $O$. taylori, para la zona.

Las nuevas gradientes altitudinales se informan para el Área Natural Protegida Parque Walter Thilo Deininger (PWTD), el 31 de agosto del 2012, localizado en el municipio de La Libertad, departamento de La Libertad (Figura 1). Forma parte del Área de Conservación Costa del Bálsamo (Fichas de Áreas de Conservación 2011). Posee ecosistemas de Bosque tropical semideciduo latifoliado de tierras bajas, bosque tropical deciduo latifoliado de tierras bajas, bosque siempreverde latifoliado aluvial de tierras bajas y bosque ripario a la largo de los ríos que atraviesan el área (Programa REDD CCAD GIZ 2011).

El segundo nuevo registro altitudinal se realizó en el Parque Nacional El Imposible (PNEI), el 3 de julio de 2007, localizado en el municipio de Tacuba, departamento de Ahuachapán (Figura 1). El sitio for- ma parte del Área de Conservación El Imposible, Barra de Santiago (Fichas de Áreas de Conservación 2011). El Área de Conservación integra ecosistemas de bosque tropical siempreverde latifoliado aluvial de tierras bajas, bosque tropical deciduo latifoliado de tierras bajas, bosque tropical semideciduo latifoliado de tierras bajas, bosque tropical semideciduo latifoliado submontano y bosque tropical semideciduo latifoliado montano inferior (Vreugdenhil et al. 2010).

Los registros disponibles en El Salvador, se encuentran resguardadas en las colecciones de referencia del Museo de Historia Natural de El Salvador (MUHNES), University of Kansas, Natural History Museum (KU), Seckemberg Museum of Franckfurt (SMF), Florida Museum of Natural History (FMNH) y Museum of Vertebrate Zoology at Berkeley (MVZ), publicados en libros y notas científicas (Brame 1968, Köhler et al. 2006, Herrera et al. 2007) (Tabla 1, Figura 1).

Los nuevos registros se realizaron durante inventarios de anfibios y reptiles en cada una de las áreas. La fase de campo en cada una de las áreas en estudio fue realizada durante la estación lluviosa para

Tabla 1. Detalles de la localización de colectas, realizadas de Oedipina taylori en El Salvador.

\begin{tabular}{|c|c|c|}
\hline Departamento & Ubicación & Código institucional \\
\hline Ahuachapán & $\begin{array}{l}\text { El Refugio, cercanías del mariposario de Francisco Serrano } \\
\text { a } 225 \text { msnm }\end{array}$ & SMF 79053 \\
\hline Ahuachapán & Parque Nacional El Imposible & MUHNES 895, 1148 \\
\hline Cabañas & Bosque de Cinquera a $400 \mathrm{msnm}$ & KU 289997 \\
\hline La Libertad & Jardín Botánico & SMF $43054^{a}$ \\
\hline La Libertad & La Joya, Ciudad Arce a 400 msnm & SMF $44392^{a}$ \\
\hline La Libertad & La Joya, Ciudad Arce a $400 \mathrm{msnm}$ & SMF $44393^{a}$ \\
\hline La Libertad & Finca El Paraíso, Santa Tecla a 900 msnm & SMF $42843^{a}$ \\
\hline La Libertad & Finca El Paraíso, Santa Tecla a 900 msnm & MVZ 40473a \\
\hline La Libertad & El Boquerón, Finca Buenos Aires a 1045msnm & SMF 44393 \\
\hline San Miguel & Volcán San Miguel & KU 291281 \\
\hline San Salvador & Parque Zoológico Nacional a $700 \mathrm{msnm}$ & SMF 81349 \\
\hline San Salvador & Ciudad de San Salvador & KU 289994a \\
\hline San Salvador & Ciudad de San Salvador & KU 61869a \\
\hline San Salvador & San Salvador a $670 \mathrm{msnm}$ & FMNH $65029^{a}$ \\
\hline San Salvador & San Salvador a $670 \mathrm{msnm}$ & FMNH $65030^{b}$ \\
\hline San Salvador & Planes de Renderos & KU $61870^{\mathrm{a}}$ \\
\hline San Salvador & Colonia San Carlos, Calle Los Naranjos & $\begin{array}{l}\text { MUHNES C-30- } \\
1342\end{array}$ \\
\hline San Vicente & Finca El Carmen & SMF 81351 \\
\hline Sonsonate & Área Natural Protegida Plan de Amayo, a 260 msnm & KUCT 11946 \\
\hline
\end{tabular}

a. Examinados por Brame 1968

b. Holotipo de Oedipina salvadorensis 


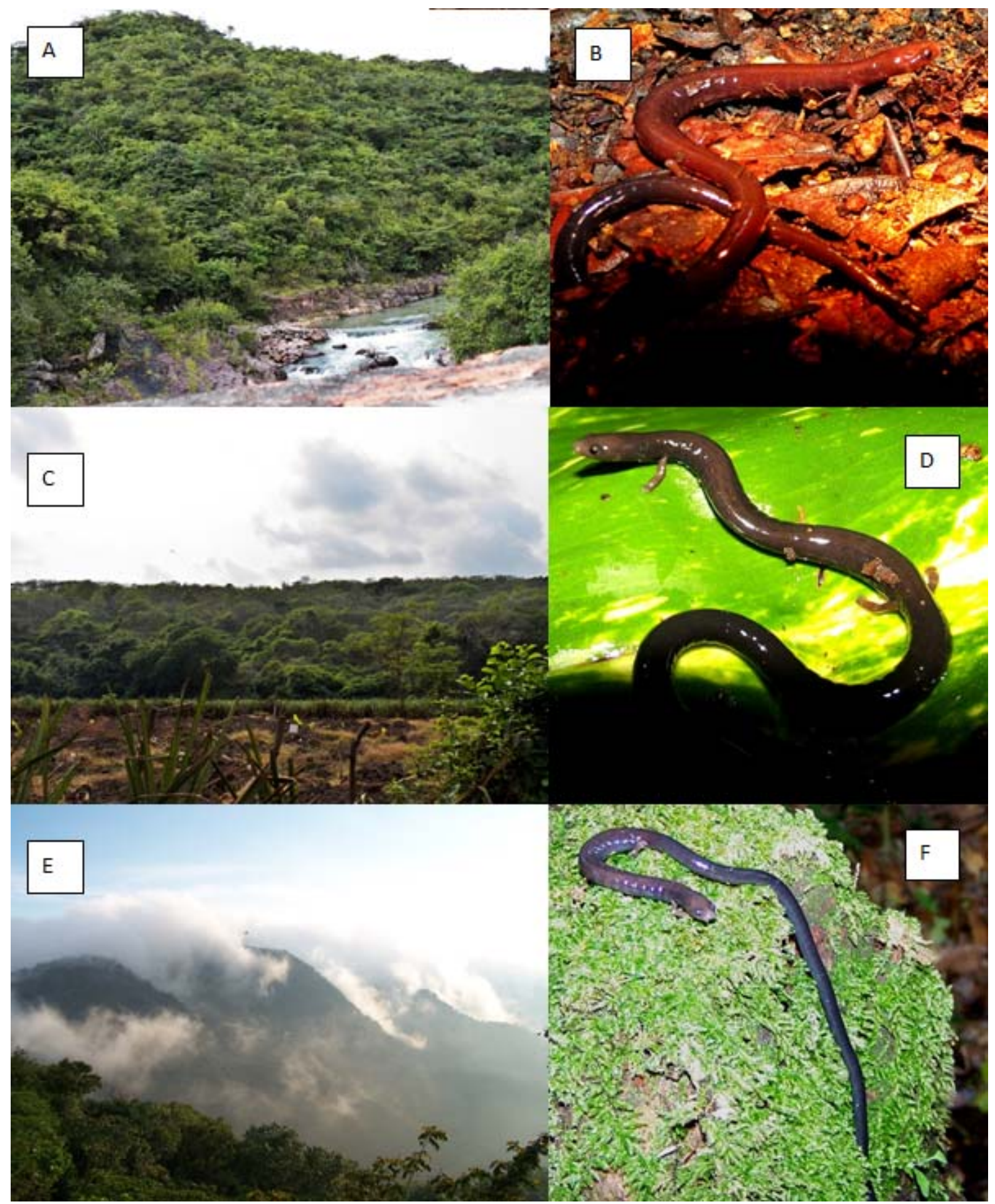

Figura. 2. Nuevo registro e individuos registrados de Oedipina taylori en el Área Natural Protegida Río Sapo, departamento de Morazán, Área Natural Protegida Parque Walter Thilo Deininger, departamento de La Libertad y Parque Nacional El Imposible departamento de Ahuachapán, El Salvador. A. Panorámica del Área Natural Protegida Río Sapo. B. Individuo de O. taylori del Área Natural Protegida Río Sapo. C. Panorámica PWTD. D. Individuo de O. taylori del PWTD. E. Panorámica del PNEI. F. Individuo de O. taylori del PNEI. 
la región. La metodología de campo utilizada fue captura manual y observación directa. Cada individuo fue fotografiado (Figura 2) e identificados en el sitio con el apoyo de las claves taxonómicas disponibles en guías de campo (Köhler et al. 2006, McCranie y Castañeda 2007, Köhler 2011). No se realizaron colectas al no contar con el permiso correspondiente.

Nuevos registros. Área Natural Protegida Río Sapo. Un individuo de O. taylori, fue encontrando a las 20:30 horas, este se encontraba bajo la corteza de un árbol caído por las lluvias, contiguo al sendero que conduce a la cabaña de los guarda-recursos del área protegida $\left(13.923459^{\circ} \mathrm{N},-88.105548^{\circ} \mathrm{W}\right.$; $666 \mathrm{msnm})$, en un sitio cubierto por vegetación siempreverde.

Parque Walter T. Deininger. Aproximadamente a las 21:30 horas, se encontró el individuo de $O$. taylori, debajo de un tronco en descomposición, en el sendero «Cueva del Encanto», que bordea una de las zonas de cultivo adyacentes al parque $\left(13.48437^{\circ}\right.$ $\mathrm{N},-89.27025^{\circ} \mathrm{W} ; 25 \mathrm{msnm}$ ). El individuo fue registrado en un área cubierta por vegetación semidecidua.

Parque Nacional El Imposible. El individuo fue encontrado a las 20:10 horas dentro de una pequeña cueva que se había formado en un paredón a un lado de un sendero ubicado en el Cerro Campana $\left(13.848847^{\circ} \mathrm{N} ;-89.906120^{\circ} \mathrm{W} ; 1275 \mathrm{msnm}\right)$. El sendero se encontraba dentro de un área cubierta por vegetación semidecidua submontana.

Esta salamandra se distribuye en una gradiente altitudinal en Guatemala desde los 140 a $1050 \mathrm{msnm}$ (Ojeda Melissa 2005-2006), El Salvador 220-1140 msnm (Köhler et al. 2006) y noroeste de Honduras 980 msnm (McCranie y Castañeda 2007), dando una gradiente altitudinal de 140-1140 msnm (Köhler 2011).

Con esta información se informan nuevas gradientes altitudinales para $O$. taylori, ya que se registró a $25 \mathrm{msnm}$ en el PWTD y a $1275 \mathrm{msnm}$ en el PNEI, agregando que no se conocen registros anteriores a este estudio, donde se reporte la presencia de la salamandra en la planicie costera de El Salvador o de otro país donde se distribuye (Ojeda Melissa 2005-2006, Köhler et al. 2006, McCranie y Castañeda 2007).

En general, O. taylori posee muy poca información sobre su distribución (McCranie y Castañeda
2007). A pesar que existe registros de su existencia en el PNEI en previas investigaciones, únicamente se había reportado su incidencia en el sector San Benito a una altura de $1148 \mathrm{msnm}$ y la zona de amortiguamiento del área protegida (Köhler et al. 2006, Acevedo et al. 2010), registrando su nueva incidencia a $1275 \mathrm{msnm}$, aumentando $135 \mathrm{~m}$ de elevación la máxima gradiente altitudinal registrada en El Salvador para esta especie. Se agrega un nuevo conocimiento de su ecología a otros ecosistemas de bosque tropical semideciduo latifoliado submontano y bosque tropical semideciduo latifoliado montano inferior (Vreugdenhil et al. 2010).

Por su ubicación geográfica, el Área Protegida Río Sapo se encuentra incluida en un ecosistema continuo, que se extiende hasta territorio de Honduras, lo que hace muy posible ampliar la distribución potencial de $O$. taylori hacia ese país, por su cercanía con la frontera, porque, según McCranie et al. (2001) y McCranie y Castañeda (2007), sólo existe un registro de $O$. taylori para Honduras, en el departamento de Francisco Morazán.

Estos nuevos registros en el Área Natural Protegida Río Sapo y el Parque Nacional El Imposible, son de gran Importancia, porque estas áreas protegidas pertenecen a las Áreas Claves para la Biodiversidad (Henríquez 2009), y contribuye a la determinación de un estatus de conservación a nivel nacional, con una distribución mejor fundamentada.

\section{Agradecimientos}

A la Agencia de los Estados Unidos para el Desarrollo Internacional (USAID), a Development Alternatives Inc. y SalvaNATURA, al cuerpo de guarda recursos del Parque Nacional El Imposible que nos acompañaron durante la investigación. Al Licenciado Antonio Escobar Macal, Jefe Unidad Centros Recreativos, del Instituto de Turismo de El Salvador (ISTU), por el permiso y para ingresar al Área Natural Protegida Walter T. Deininger. A René Eduardo Urrutia por el apoyo en la fase de campo, en el PWTD.

\section{Literatura citada}

Acevedo M, Wake D, Köhler G. 2010. Oedipina taylori. In: IUCN 2012. IUCN Red List of Threatened Species. 
Version 2012.2. [Consultado el 15 de mayo 2013]. Electronic data base. http://www.iucnredlist.org

Boza-Oviedo E, Rovito SM, Chaves G, et al. 2012. Salamanders from the eastern Cordillera de Talamanca, Costa Rica, with descriptions of five new species (Plethodontidae: Bolitoglossa, Nototriton, and Oedipina) and natural history notes from recent expeditions. Zootaxa. 3309: 3661.

Brame A. 1968. Systematics and evolution of the Mesoamerican salamander genus Oedipina. J Herpetol. 2: 1-64.

Brodie EDJr, Campbell Y, Jonathan A. 1993. A new salamander of the genus Oedipina (Caudata: Plethodontidae) from the Pacific versant of Guatemala. J Herpetol. 49 (2): 25965.

Brodie EDJr, Acevedo ME, Campbell JA. 2012. New salamanders of the genus Oedipina (Caudata: Plethodontidae) from Guatemala. J Herpetol. 46: 233-40.

Centro de Estudios Ambientales y Biodiversidad, Alianza para la Conservación de Bosques de Pino-Encino de Mesoamérica, The Nature Conservancy. 2009. Diagnóstico ecológico y socioeconómico de la ecorregión bosques de pino-encino de Centroamérica. Guatemala: The Nature Conservancy/Universidad del Valle de Guatemala. 335 pp.

Fichas de Áreas de Las Conservación. 2011. Estudio de racionalización y priorización del sistema de áreas naturales protegidas de la República de El Salvador. San Salvador: Ministerio de Medio Ambiente y Recursos Naturales. Elaborado por el World Institute for Conservation and Environment. 258 pp.

García-París M, Good M, Parra-Olea G, Wake DW. 2000. Biodiversity of Costa Rica salamanders: implications of high levels of genetic differentiation and phylogegraphic structure for species formation. Proceed Nat Acad Sci USA. 97: 1640-47.

Henríquez V. 2009. Las áreas de importancia para la conservación de la biodiversidad. El Salvador: salvaNATURAS. $32 \mathrm{pp}$.

Herrera N, Henríquez V, Greenbaum E. 2007. New country and department records for amphibians and reptiles from El Salvador. Herpetol Rev. 38 (2): 222-6.

Köhler G. 2011. Amphibians of Central America. Offenbach: Herpeton Verlag. 376 pp.

Köhler G, Veselý M, Greenbaun E. 2006. The amphibians and reptiles of El Salvador. Malabar: KriegerPubl Co. 238 pp.

Leenders TA, Watkins-Colwell YGJ. 2004. Notes on a collection of amphibians and reptiles from El Salvador. New Haven: Peabody Museum of Natural History, Yale University. 33 pp.
McCranie JR, Cruz GA, Rivera DM. 2001. Geographic distribution. Oedipina taylori. Herpetol Rev. 32 (1): 54.

McCranie, JR, Castañeda, EF. 2007. Guía de campo de anfibios de Honduras. Salt Lake City: Bibliomania. 304 pp.

McCranie JR, Townsend JH. 2011. Description of a new species of worm salamander (Caudata, Plethodontidae, Oedipina) in the subgenus Oedopinola from the central portion of the Cordillera Nombre de Dios, Honduras. Zootaxa. 2990: 59-68.

Misterio de Medio Ambiente Recursos Naturales (MARN). 2009. Listado oficial de especies de vida silvestre, amenazadas o en peligro de extinción. Acuerdo $\mathrm{N}^{\mathrm{o}} 36$. San Salvador: Diario Oficial N¹02, Tomo N³83.

Ministerio de Medio Ambiente y Recursos Naturales (MARN). 2011. Estudio de racionalización y priorización del sistema de áreas naturales protegidas de la República de El Salvador. San Salvador: World Institute for Conservation and Environment. 254 pp.

Ojeda M. 2005-2006. Salamandras endémicas para la región de Guatemala. Programa EDC-integrado. Guatemala: Escuela de Biología, Universidad de San Carlos de Guatemala. 43 pp.

Programa REDD-CCAD-GIZ. 2011. Tipos de bosque y contexto del mapeo de la cobertura forestal en Centro América y República Dominicana. Antigua: Deutsche Gesellsachaft für Internationale Zusammenarbeit. 18 pp.

Rand AS. 1952. A new salamander of the genus Oedipina from El Salvador. Nat Hist Miscell. 98: 1-3.

Rand AS. 1957. Notes on amphibians and reptiles from El Salvador. Nat Hist Miscell. 34 (42): 1-44.

Stuart LC. 1952. Some new amphibians from Guatemala. Proceed Biol Soc Washington. 65: 1-12.

Sunyer J, Townsend JH, Wake DB, Travers SL, González SC, Obando LA, Quintana AZ. 2011. A new cryptic species of salamander, genus Oedipina (Caudata: Plethodontidae), from premontane elevations in northern Nicaragua, with comments on the systematic status of the Nicaraguan paratypes of O. pseudouniformis Brame, 1968. Breviora. Mus Comparat Zool. 526: 1-16.

Taylor EH. 1952. The salamanders and caecilians of Costa Rica. Sci Bull. 34: 695-791.

Vreugdenhil D, Machado M, Linares J, Henríquez Cisneros VE. 2011. Líneas estratégicas para la racionalización del sistema de las Áreas Naturales Protegidas de El Salvador. San Salvador: WICE.

Wake BD, Good AD. 1997. Phylogenetic and taxonomic implications of protein variation in the Mesoamerican salamander genus Oedipina (Caudata: Plethodontidae). Rev Biol Trop. 45 (3): 85-1208. 